\title{
Duration of antibiotic therapy in systemic lupus erythematosus patients with hospital-acquired bacterial pneumonia in eastern China
}

\author{
Cheng Zhu ${ }^{1 \#}$, Yujie $\mathrm{Li}^{2 \#}$, Yuetian $\mathrm{Yu}^{2}$, Liangjing $\mathrm{Lu}^{3}$ \\ ${ }^{1}$ Department of Disease Prevention and Control, Ruijin Hospital, Shanghai Jiao Tong University, School of Medicine, Shanghai, China; ${ }^{2}$ Department \\ of Critical Care Medicine, Ren Ji Hospital, School of Medicine, Shanghai Jiao Tong University, Shanghai, China; ${ }^{3}$ Department of Rheumatology, \\ Ren Ji Hospital, School of Medicine, Shanghai Jiao Tong University, Shanghai, China \\ Contributions: (I) Conception and design: C Zhu, Y Yu; (II) Administrative support: L Lu; (III) Provision of study materials or patients: Y Li, Y Yu; (IV) \\ Collection and assembly of data: C Zhu, Y Yu; (V) Data analysis and interpretation: C Zhu, Y Yu; (VI) Manuscript writing: All authors; (VII) Final \\ approval of manuscript: All authors. \\ "These authors contributed equally to this work. \\ Correspondence to: Yuetian Yu. Department of Critical Care Medicine, Ren Ji Hospital, School of Medicine, Shanghai Jiao Tong University, Shanghai \\ 200001, China. Email: fishyyt@sina.com; Liangjing Lu. Department of Rheumatology, Ren Ji Hospital, School of Medicine, Shanghai Jiao Tong \\ University, Shanghai 200001, China. Email: lu_liangjing@163.com.
}

Background: To investigate the duration of antibiotic therapy and its influencing factors in treating systemic lupus erythematosus (SLE) patients with hospital-acquired bacterial pneumonia (HABP).

Methods: Clinical data of SLE patients with HABP from January 2015 to December 2019 were collected. Duration of antibiotic treatment and its correlations with the severity of pneumonia, status of SLE and the time to clinical stability (TCS) were analysed. A logistic regression model was performed to screen the risk factors influencing the total antibiotic treatment course $>7$ days.

Results: Three hundred and forty-four patients were finally enrolled in the study and the mean duration of total antibiotic therapy was 9.5 days. The TCS was related to the duration of antibiotic therapy $\left(\mathrm{R}^{2}=0.76\right.$, $\mathrm{P}<0.0001)$. More patients with multidrug-resistant pathogen infection and Candida colonization were found in the total duration $>7$ days group. SLE disease activity index (SLEDAI) [odds ratio (OR) $=5.52,95 \%$ confidence interval (CI): 3.59-7.28], taking immunosuppressants during HABP treatment (OR =5.29, 95\% CI: 3.93-7.09) and multidrug-resistant pathogen infection (OR =4.91, 95\% CI: 1.45-6.87) were greatly impacted risk factors $(\mathrm{P}<0.05$, respectively).

Conclusions: The duration of antibiotic therapy in SLE patients with HABP was longer than the course recommended by practice guidelines. The severity of the disease and the host's immune status might influence the duration of treatment.

Keywords: Hospital-acquired bacterial pneumonia (HABP); systemic lupus erythematosus (SLE); immunocompromised host (ICH); antibiotics

Submitted Mar 08, 2020. Accepted for publication Dec 22, 2020.

doi: 10.21037/apm-20-584

View this article at: http://dx.doi.org/10.21037/apm-20-584

\section{Introduction}

Infectious disease is among the leading causes of mortality in immunocompromised hosts (ICHs) especially in those who are diagnosed with systemic lupus erythematosus (SLE).
As reported, about thirty percent of these patients present with infections during follow-up and more than one-third of deaths are highly correlated with these diseases (1). Among these infectious diseases, hospital-acquired bacterial 
pneumonia (HABP) accounts for the vast majority of cases due to respiratory tract dissemination, immunosuppressive agent treatment and glucocorticoid usage (2).

Practice guidelines for the management HABP have been published to direct the empirical antibiotic therapy and the de-escalation strategy $(3,4)$. However, almost all the guidelines do not cover the ICHs like those with SLE $(5,6)$. Thus, information from the current literature about these patients is limited. Despite the enormous progress in the field of HABP management in ICHs, mortality has not decreased substantially in the last decade (2). Many questions remain unanswered about the treatment such as the appropriate duration of antibiotic treatment. The recommended antimicrobial treatment course was shortened from two weeks to approximately seven days (7). Whether "shorter is better" is still unknown, especially in immunocompromised patients with HABP.

Therefore, this retrospective cohort study was conducted to analysis the following status: (I) the course of antibiotic treatment for SLE patients with HABP and (II) factors that influence clinicians' decisions about the duration of antibiotic therapy.

\section{Methods}

\section{Research briefs}

The retrospective cohort study was conducted in Rui Jin and Ren Ji Hospital, which are the top two pneumonia and autoimmune disease management medical institutions in eastern China. Information that needs to be analysed was obtained from each hospital's electronic medical records between January 2015 and December 2019 by three attending physicians. Ethics committee of Shanghai Jiaotong University School of Medicine approved the study (No. 2017-201) and it was conducted in accordance with the Declaration of Helsinki (as revised in 2013). The written informed consent was obtained from all patients.

\section{Study population}

In patients who were diagnosed with SLE were included in the study if they were (I) suspected of HABP and (II) within the range of 18 and 80 years old. In patients were excluded if they (I) had an extrapulmonary infection; (II) had no positive pathogen isolation results by culture; (III) died while the duration of antibiotic treatment was not finished; (IV) were pregnant and (V) had an incomplete medical history.

\section{Disease and parameter definitions}

The criteria of HABP definition based on the clinical practice guideline by Infectious Diseases Society of America were listed as follows: (I) new infiltrate could be found on patients' pulmonary imaging $48 \mathrm{~h}$ after hospital admission; (II) bacteria could be isolated from the samples of respiratory tract secrets and (III) at least two of the following criteria could be met: (i) leucocytosis $\geq 10 \times 10^{9} / \mathrm{L}$ or $\leq 4 \times 10^{9} / \mathrm{L}$; (ii) an abnormal temperature $\left(>37.8\right.$ or $\left.<35.6^{\circ} \mathrm{C}\right)$; and (iii) newly purulent sputum (3). The course of antibiotic treatment was defined as antibiotic administration for treating HABP both during the hospital stay and after hospital discharge (either intravenous or oral prescription).

According to the guideline of American Thoracic Society (8), the criteria for clinical stability were defined as follows: (I) lack of fever for $>8 \mathrm{~h}$; (II) improvement in clinical signs (cough and shortness of breath); (III) improvement in leucocytosis (decrease $>10 \%$ from the previous day); and (IV) tolerance of oral antibiotic intake. The time to clinical stability (TCS) was calculated as the number of days from the date of hospital admission to the date when the patient met the clinical stability criteria (9).

\section{Data collection}

Clinical data about the enrolled patients was made up of five parts: (I) basic characteristics like age, gender and comorbidity; (II) status of SLE, including the SLE disease activity index (SLEDAI) and the concentration of complement; (III) information about the host immune status and treatment, including daily prednisone dose, administration of immunosuppressant; (IV) antibiotics treatment and $(V)$ the level of inflammatory factors.

\section{Identification of strains and antimicrobial susceptibility testing operation}

Bacteria that caused HABP of patients with SLE and the results of antimicrobial susceptibility testing were obtained from the microbiological database of the laboratory. Pathogens from lower respiratory tract specimens were identified using a bioMérieux VITEK-2 automated system. The results of antimicrobial susceptibility testing were determined according to M100-S29 (10). 


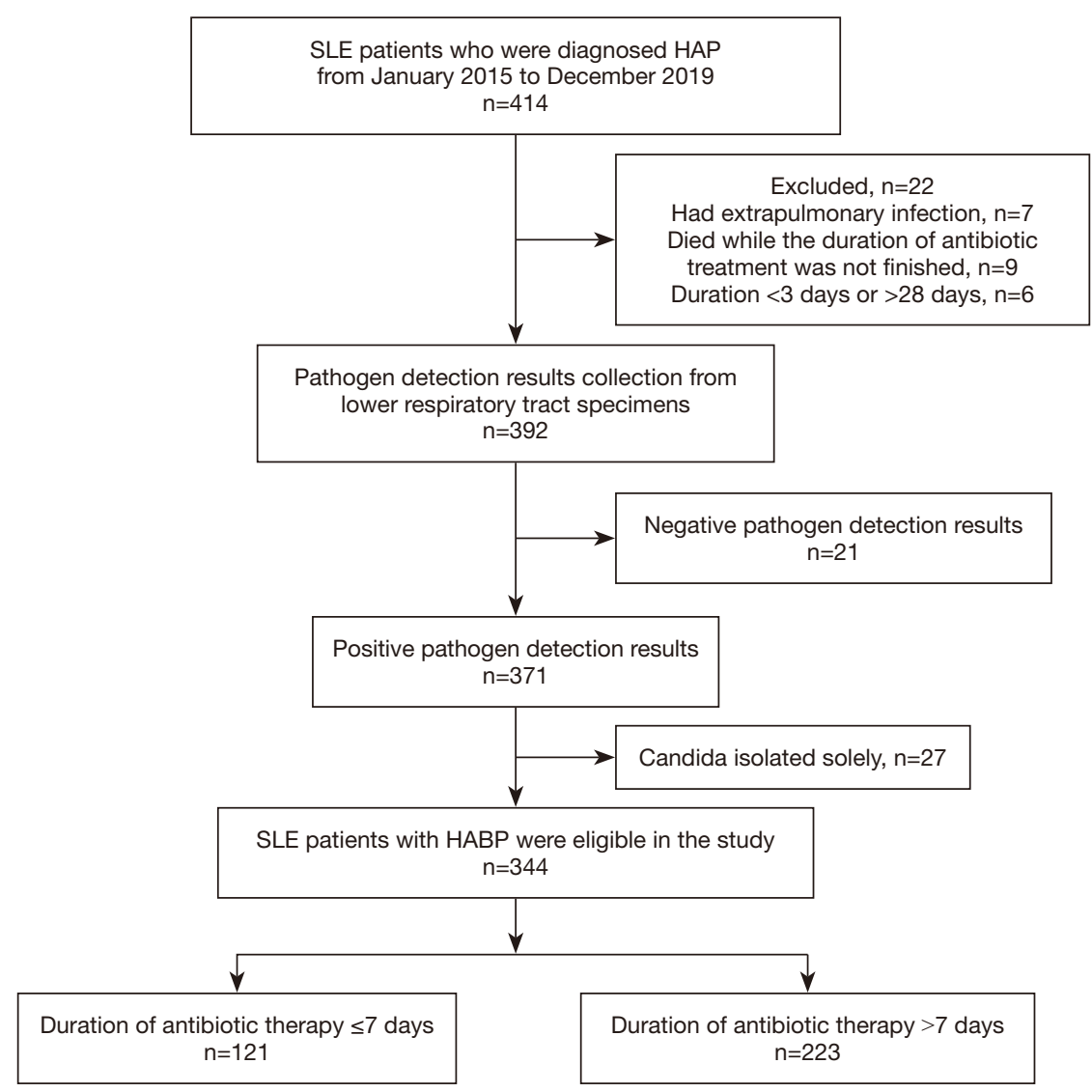

Figure 1 Flow chart of the study. SLE patients with HABP enrolled in the study. HAP, hospital-acquired pneumonia; HABP, hospitalacquired bacterial pneumonia; SLE, systemic lupus erythematosus.

\section{Statistical analysis}

SPSS version 21.0 was applied to perform the statistical analysis. Chi-square test was applied for equal proportions and the results were presented as percentages (n). Normally distributed variables were compared by Student's t-test and the results were expressed as the means \pm standard deviations. Factors related to prolonged duration of antibiotic therapy for more than 7 days were screened by logistic regression and revealed as odds ratios (ORs) and $95 \%$ confidence intervals (CIs).

\section{Results}

\section{Study population}

In total, 414 immunocompromised patients were the initial screened in each hospital's medical record system and 344 of them were finally included. Among the study population,
$223(64.8 \%)$ had a duration of antibiotic therapy $>7$ days, while the other 121 had a duration of antibiotic therapy $\leq 7$ days (Figure 1).

\section{Causative pathogens and duration of antibiotic therapy}

More than 10 bacteria strains were isolated from the respiratory secretions of HABP patients. Non-fermentative gram-negative bacilli ( $\mathrm{n}=178$ ) was the most commonly responsible organism and accounted for approximately half of cases $(51.7 \%)$. In total, three quarters of the HABP were caused only by gram-negative organisms $(n=261,75.9 \%)$ while others were caused only by gram-positive organisms $(\mathrm{n}=83,24.1 \%)$. Among the strains isolated, 58 (16.8\%) were identified as MDR pathogens, and Candida colonization with bacterial infection was found in $110(31.9 \%)$ patients (Figure 2A). The mean cause of total antibiotic treatment was 9.5 days, which exceeded the recommended course 
A

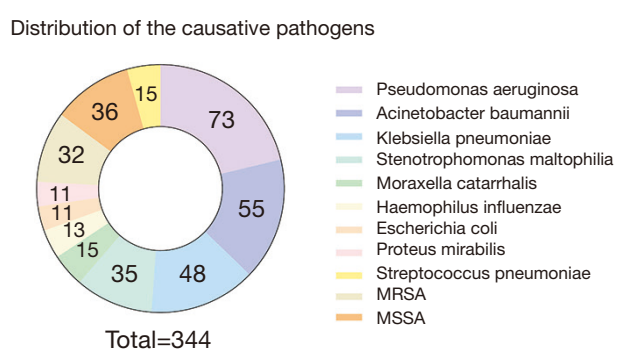

B

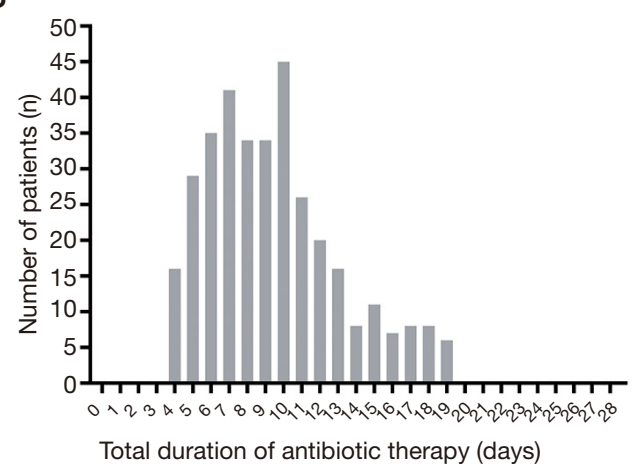

Figure 2 Causative pathogens and duration of antibiotic therapy. (A) Distribution of the causative pathogens. (B) The duration of antibiotic therapy. MRSA, methicillin resistant Staphylococcus aureus; MSSA, methicillin sensitive Staphylococcus aureus.
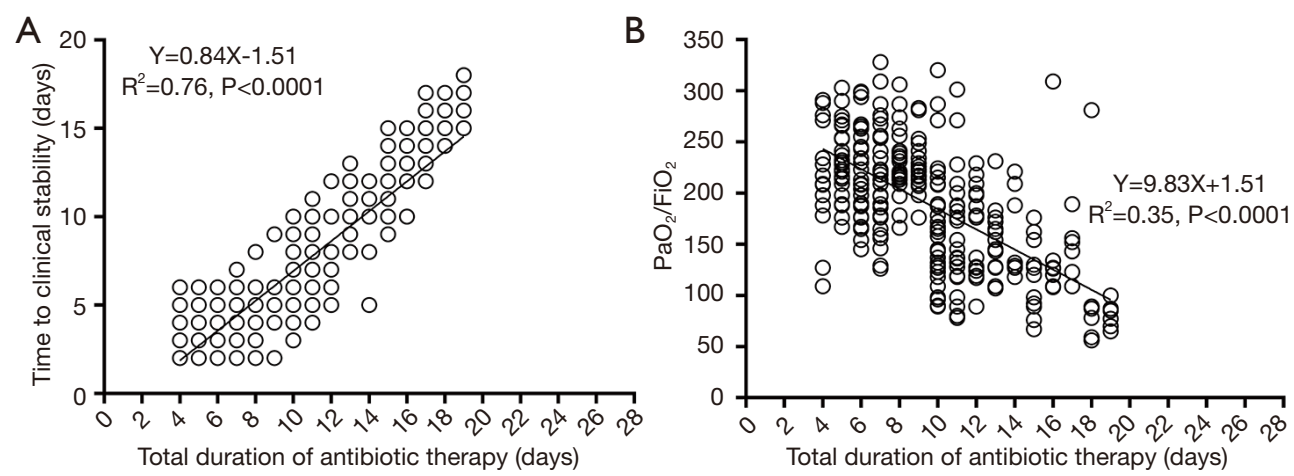

Figure 3 Correlation of severity of HABP, clinical response and duration of antibiotic therapy. (A) A relationship was found between the time to clinical stability and the total duration of antibiotic therapy. (B) A relationship was found between the $\mathrm{PaO}_{2} / \mathrm{FiO}_{2}$ and the total duration of antibiotic therapy. HABP, hospital-acquired bacterial pneumonia.

(7 days) set forth in the HABP guidelines (Figure 2B).

\section{Severity of HABP, clinical response and duration of antibiotic therapy}

The mean TCS was $6.5 \pm 3.5$ days in SLE patients with HABP in our study. The relationship between antibiotic treatment duration and TCS was listed in Figure 3A. A linear relationship was found between the total duration of antibiotic therapy and TCS $\left(\mathrm{R}^{2}=0.76, \mathrm{P}<0.0001\right)$ as well as the total duration of antibiotic therapy and $\mathrm{PaO}_{2} / \mathrm{FiO}_{2}$ $\left(\mathrm{R}^{2}=0.35, \mathrm{P}<0.0001\right)$ (Figure $3 B$ ).

\section{Clinical features of the total duration $>7$ days and $\leq 7$ days groups}

The demographics and clinical characteristics of the
223 patients with the total duration $>7$ days and the 121 patients with the total duration $\leq 7$ days are listed in Table 1 . Female patients $(91.6 \%)$ made up the vast majority of study population. More patients with MDR pathogen infection and Candida colonization were found in the total duration $>7$ days group. Furthermore, a relatively higher levels of inflammatory factors were indicated in the same group (Table 1).

As for SLE status, more patients taking a high dose of daily prednisone $(60.9 \%$ vs. $28.1 \%)$ or receiving immunosuppressant treatment during HABP therapy $(14.8 \%$ vs. $6.6 \%)$ were found in the total duration $>7$ days group, together with a higher SLEDAI $(\mathrm{P}<0.05$, respectively). No difference was found in the count of natural killer cell or B lymphocyte cell between the two groups, $\mathrm{P}>0.05$ (Table 2).

In terms of antimicrobial use, $\beta$-lactam $/ \beta$-lactamase inhibitor was the most common choice, which accounted 
Table 1 Demographics and clinical characteristics of HABP patients with SLE

\begin{tabular}{|c|c|c|c|}
\hline Characteristics & Total duration $>7$ days $(n=223)$ & Total duration $\leq 7$ days $(\mathrm{n}=121)$ & $P$ value \\
\hline Female, n (\%) & $207(92.8)$ & $108(89.3)$ & 0.255 \\
\hline Respiratory rate & $30.6 \pm 4.8$ & $31.4 \pm 5.9$ & 0.175 \\
\hline $\mathrm{PaO}_{2} / \mathrm{FiO}_{2}<200$ mmHg, n (\%) & $139(62.3)$ & $29(23.9)$ & 0.001 \\
\hline White blood cells, $10^{9} / \mathrm{L}$ & $15.6 \pm 3.3$ & $16.2 \pm 2.9$ & 0.094 \\
\hline Neutrophil, \% & $83.1 \pm 7.3$ & $82.5 \pm 7.4$ & 0.469 \\
\hline C-reactive protein, $\mathrm{mg} / \mathrm{L}$ & $122.6 \pm 23.8$ & $114.8 \pm 17.8$ & 0.002 \\
\hline Procalcitonin, ng/mL & $3.9 \pm 0.7$ & $3.7 \pm 0.8$ & 0.017 \\
\hline Immunoglobulin A, g/L & $3.42 \pm 0.73$ & $3.29 \pm 0.58$ & 0.092 \\
\hline Urea, mmol/L & $7.5 \pm 1.4$ & $7.3 \pm 1.3$ & 0.196 \\
\hline Creatinine clearance, $\mathrm{dL} / \mathrm{min}$ & $1.7 \pm 0.6$ & $2.5 \pm 0.5$ & 0.001 \\
\hline MDR pathogen infection, $\mathrm{n}(\%)$ & $53(23.8)$ & $17(14.0)$ & 0.033 \\
\hline Candida colonization with bacterial infection, $\mathrm{n}(\%)$ & $94(42.2)$ & $22(18.2)$ & 0.001 \\
\hline
\end{tabular}

HABP, hospital-acquired bacterial pneumonia; SLE, systemic lupus erythematosus; MDR, multidrug-resistant.

Table 2 SLE status of the patients with HABP

\begin{tabular}{|c|c|c|c|}
\hline Characteristics & Total duration $>7$ days $(n=223)$ & Total duration $\leq 7$ days $(n=121)$ & $P$ value \\
\hline \multicolumn{4}{|l|}{ SLE activity at the time of HABP, $n(\%)$} \\
\hline Lupus nephritis & $54(24.2)$ & $18(14.9)$ & 0.042 \\
\hline Hematological activity & $22(9.9)$ & $10(8.3)$ & 0.625 \\
\hline Daily prednisone dose $>0.5$ mg/kg/d, n (\%) & $136(60.9)$ & $34(28.1)$ & 0.001 \\
\hline Taking immunosuppressants during HABP treatment, $\mathrm{n}(\%)$ & $33(14.8)$ & $8(6.6)$ & 0.025 \\
\hline CD4/CD8 ratio <1, n (\%) & $142(63.7)$ & $55(45.5)$ & 0.001 \\
\hline Natural killer cell, cells/uL & $136.4 \pm 53.7$ & $145.3 \pm 41.8$ & 0.115 \\
\hline Ts lymphocyte, cells/uL & $211.8 \pm 58.2$ & $208.9 \pm 61.3$ & 0.665 \\
\hline Positive anti-dsDNA, n (\%) & $146(65.5)$ & $86(71.1)$ & 0.289 \\
\hline Concentration of $\mathrm{C} 3, \mathrm{~g} / \mathrm{L}$ & $0.5 \pm 0.1$ & $0.5 \pm 0.2$ & 1.000 \\
\hline SLEDAI score & $7.4 \pm 2.3$ & $5.1 \pm 2.6$ & $<0.001$ \\
\hline
\end{tabular}

HABP, hospital-acquired bacterial pneumonia; SLE, systemic lupus erythematosus; SLEDAl, systemic lupus erythematosus disease activity index. 
Table 3 Antibiotics prescription of the SLE patients with HABP

\begin{tabular}{|c|c|c|c|}
\hline Treatment & Total duration $>7$ days $(n=223), n(\%)$ & Total duration $\leq 7$ days $(n=121), n(\%)$ & $P$ value \\
\hline Carbapenem & $87(39.0)$ & $31(25.6)$ & 0.012 \\
\hline Quinolones & $45(20.2)$ & $27(22.3)$ & 0.642 \\
\hline Third generation cephalosporins & $32(14.3)$ & $17(14.0)$ & 0.939 \\
\hline$\beta$-lactam $/ \beta$-lactamase inhibitors & $93(41.7)$ & $48(39.7)$ & 0.714 \\
\hline Linezolid & $18(8.1)$ & $12(9.9)$ & 0.562 \\
\hline Vancomycin & $19(8.5)$ & $10(8.3)$ & 0.935 \\
\hline Antibiotics double or triple therapy & $118(52.9)$ & $31(25.6)$ & 0.001 \\
\hline
\end{tabular}

HABP, hospital-acquired bacterial pneumonia; SLE, systemic lupus erythematosus.

for almost half the prescriptions. More patients in the total duration $>7$ days group received double or triple antibiotics therapy $(52.9 \%$ vs. $25.6 \%)$ and antimicrobial de-escalation treatment $(41.3 \%$ vs. $9.9 \%)$ was found more commonly in the same group $(\mathrm{P}<0.001$, respectively) (Table 3).

\section{Influencing factors of the total duration $>7$ days}

Based on the multivariate logistic regression analysis, SLEDAI $(\mathrm{OR}=5.52,95 \% \mathrm{CI}: 3.59-7.28)$, taking immunosuppressants during HABP treatment $(\mathrm{OR}=5.29$, 95\% CI: 3.93-7.09) and MDR pathogen infection $(\mathrm{OR}=4.91$, 95\% CI: 1.45-6.87) were the factors that might influence the duration $(\mathrm{P}<0.05$, respectively). Although more patients with septic shock were found in the total duration $>7$ days group than in the $\leq 7$ days group ( $13.0 \%$ vs. $5.8 \%, \mathrm{P}=0.037)$, it could not be confirmed as a factor influencing the antibiotic treatment course (Figure 4).

\section{Discussion}

In this retrospective observational study, we analysed the duration of antibiotic treatment in SLE patients with HABP who were not addressed in the practice guidelines. We found that the mean cause of total antibiotic treatment was 9.5 days, which exceeded the recommended course.

SLEDAI, taking immunosuppressants during HABP treatment and MDR pathogen infection were the top three risk factors.
HABP is the leading causes of mortality in ICHs worldwide and is caused by numerous microorganisms $(4,5)$. Thus, all efforts should be focused on preventing and properly treating the disease, but appropriate treatment of HABP in SLE patients is quite challenging because of the limited data available regarding the duration of antimicrobial therapy.

Based on our study, non-fermentative gram-negative bacilli is the most common organism responsible for approximately half of cases. Other common pathogens include Klebsiella pneumoniae and Moraxella catarrhalis, which are similar to the pathogens associated with HABP in the general adult population of China (11). However, there are more patients with MDR pathogen infections and Candida colonization in our study, which might be caused by long-term antimicrobial exposure or glucocorticoid treatment (12).

As recommended by practice guidelines, a duration of 7 days is appropriate for most HABP with low or moderate severity. What needs to be emphasized is that all the recommendations above are suitable for the non- $\mathrm{ICH}$. Our study revealed that the mean duration of total antibiotic therapy of hospitalized immunocompromised patients with HABP was 9.5 days and more than half patients had a duration of antibiotic therapy $>10$ days.

The severity of pneumonia on admission and the clinical stability of the patients are two dominant factors that guide clinicians to determine when to initiate antibiotic therapy and when to discontinue it. $\mathrm{PaO}_{2} / \mathrm{FiO}_{2}$ can reflect the degree of hypoxia in patients with pneumonia. If the 


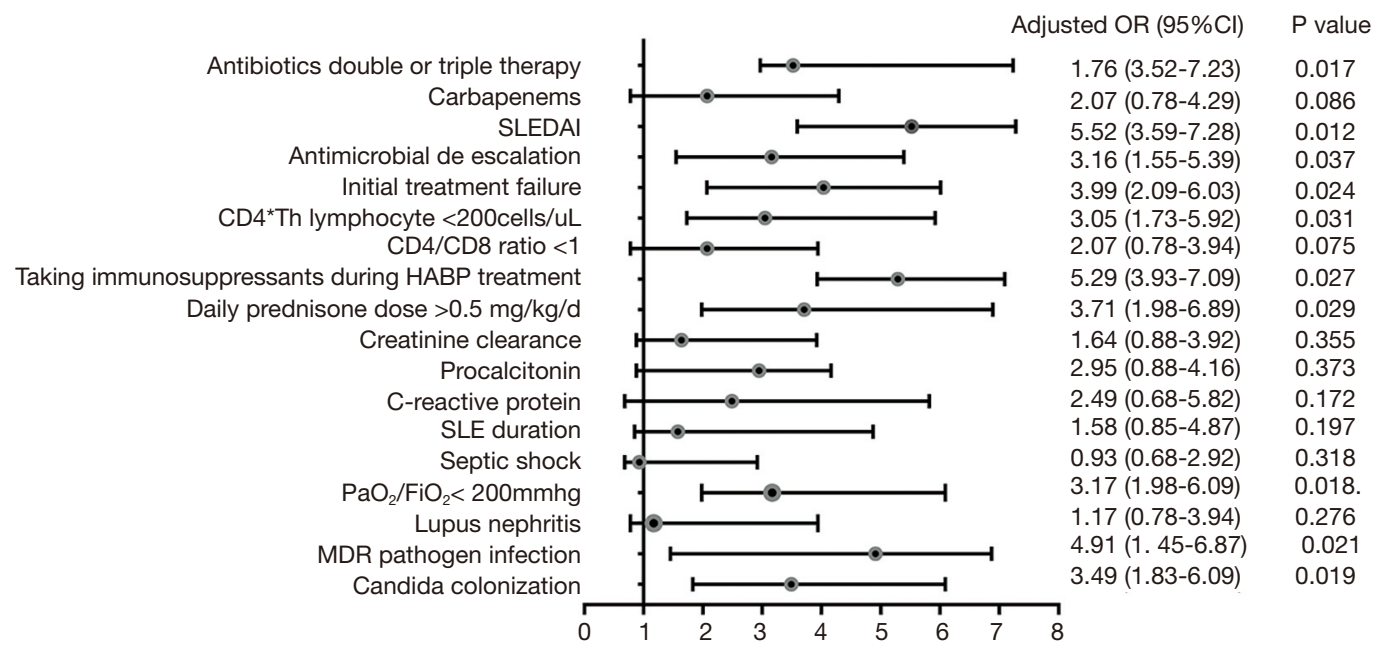

Figure 4 Multivariate logistic regression analysis of risk factors for total antibiotic duration $>7$ days. SLEDAI, taking immunosuppressants during HABP treatment, MDR pathogen infection were the top three risk factors as well as the other 7 risk factors. Septic shock was suggested proven to be statistically nonsignificant. SLE, systemic lupus erythematosus; SLEDAI, systemic lupus erythematosus disease activity index; MDR, multidrug-resistant; HABP, hospital-acquired bacterial pneumonia.

value is less than $200 \mathrm{mmHg}$, it means that the patients is in a moderate to severe condition. Therefore, a correlation between the total duration of treatment and $\mathrm{PaO}_{2} / \mathrm{FiO}_{2}$ was found in our study, which was consistent with Aliberti's research (13). What is more, the administration of double or triple antibiotic therapy was easily identified in these patients as well as in patients with MDR pathogen infection. There was a longer period from hospital admission to TCS and more de-escalation therapy was noted, both of which prolonged the duration of antibiotic therapy.

Clinical responses to antibiotic treatment and the TCS are based on host immune status, pathogen features and antibiotic characteristics (14). The vast majority of patients enrolled in our study had decreased CD4+Th lymphocyte cell counts, which indicated the defective host immune status and prolonged antibiotic treatment duration. It was also noted that patients were treated with two or three extra days of antibiotic therapy beyond the TCS, in case of HABP reoccurrence. Therefore, a correlation was found between TCS and the total duration of antibiotic treatment.

Glucocorticoid prescription or immunosuppressant treatment are not uncommon in immunocompromised patients, and both can disrupt the immune system. A reduction in CD4+Th lymphocyte cell counts and a decreased CD4+Th/CD8+Th ratio can occur during the course of primary disease treatment as well as a deficiency in the components of the complement system $(15,16)$. All these actions will result in weakening the normal defences of the body and favouring the aggression of microorganisms. Thus, the duration of antibiotic therapy was prolonged, and daily prednisone dose $>0.5 \mathrm{mg} / \mathrm{kg} / \mathrm{d}$ as well as taking immunosuppressants during HABP treatment were proven to be the risk factors.

The isolation of Candida species from respiratory secretions is common. In most cases, it is always judged colonization, and antifungal treatment is rarely necessary (17). However, the co-existence of fungi and bacteria has attracted considerable attention in the last decade. In vitro experiments have shown that Candida and MDR pathogens are both capable of utilizing arachidonic acid to form eicosanoids, which might affect the pharmacokinetics of antibiotics (18). In Roux's study, it was noted that airway fungal colonization could elicit a Th1-Th17 immune response that favoured the development of bacterial pneumonia (19). Furthermore, scanning electron microscopy showed that bacteria can attach tightly to the surface of Candida albicans filaments, which led to the prolonged application of antibiotics (20).

In our study population, Candida colonization was identified in 116 HABP patients, and the total duration of antibiotic therapy was longer than 7 days. As was demonstrate in our study, Candida colonization was an independent risk factor that predicted the prolonged course of antibiotic treatment. Thus, special attention should be paid to these patients. In addition, further research is 
needed to determine whether HABP patients with Candida colonization would benefit from anti-fungal therapy.

SLEDAI contains 24 items which can reflect the activity of SLE. A higher SLEDAI could also promote the infectious diseases such as HABP because of the complement consumption (12). SLEDAI $>6$ indicates that the status of SLE is in the mild to moderate active condition which leads to more glucocorticoid prescription. Thus, it was proved to be an important risk factor to prolong the antibiotic therapy.

For the retrospective study, we were mainly concerned with SLE patients with HABP. However, there are still many unresolved issues in the research. Both duration and doses of antibiotic therapy are important for clinical decision making. In our study, therapeutic drug monitoring (TDM) of antibiotics could not be applied during the whole period of hospitalization. Numerous studies have found that hemodynamic instability, altered fluid balance, organ disfunction and organ support are the main factors that affect the concentration of antibiotics (21-23). Therefore, it will also have a further impact on the duration of antibiotic therapy which cannot be neglected. In addition, physicians' medical training on antibiotic therapy and the hospital's uniform requirement on antibiotic use are also important for the decision of anti-infection treatment. A further analysis of the factors for decision making should be performed in the future.

\section{Conclusions}

The present investigation assessed the duration of antibiotic therapy in SLE patients with HABP and found that it was longer than the course recommended by practice guidelines. The severity of the disease and the host's immune status might influence the duration of treatment. More prospective studies and randomized controlled trials should be performed for subsequent analysis.

\section{Acknowledgments}

Funding: This work was supported by the National Key Research and Development Program of China (2017YFC0909002) and the Scientific Research Project of Shanghai Municipal Health Bureau (201840006).

\section{Footnote}

Data Sharing Statement: Available at http://dx.doi. org/10.21037/apm-20-584
Conflicts of Interest: All authors have completed the ICMJE uniform disclosure form (available at http://dx.doi. org/10.21037/apm-20-584). The authors have no conflicts of interest to declare.

Ethical Statement: The authors are accountable for all aspects of the work in ensuring that questions related to the accuracy or integrity of any part of the work are appropriately investigated and resolved. Ethics committee of Shanghai Jiaotong University School of Medicine approved the study (No. 2017-201) and it was conducted in accordance with the Declaration of Helsinki (as revised in 2013). The written informed consent was obtained from all patients.

Open Access Statement: This is an Open Access article distributed in accordance with the Creative Commons Attribution-NonCommercial-NoDerivs 4.0 International License (CC BY-NC-ND 4.0), which permits the noncommercial replication and distribution of the article with the strict proviso that no changes or edits are made and the original work is properly cited (including links to both the formal publication through the relevant DOI and the license). See: https://creativecommons.org/licenses/by-nc-nd/4.0/.

\section{References}

1. Danza A, Ruiz-Irastorza G. Infection risk in systemic lupus erythematosus patients: susceptibility factors and preventive strategies. Lupus 2013;22:1286-94.

2. García-Guevara G, Rios-Corzo R, Diaz-Mora A, et al. Pneumonia in patients with systemic lupus erythematosus: Epidemiology, microbiology and outcomes. Lupus 2018;27:1953-9.

3. Kalil AC, Metersky ML, Klompas M, et al. Management of Adults With Hospital-acquired and Ventilator-associated Pneumonia: 2016 Clinical Practice Guidelines by the Infectious Diseases Society of America and the American Thoracic Society. Clin Infect Dis 2016;63:e61-e111.

4. Torres A, Niederman MS, Chastre J, et al. International ERS/ESICM/ESCMID/ALAT guidelines for the management of hospital-acquired pneumonia and ventilator-associated pneumonia: Guidelines for the management of hospital-acquired pneumonia (HAP)/ ventilator-associated pneumonia (VAP) of the European Respiratory Society (ERS), European Society of Intensive Care Medicine (ESICM), European Society of Clinical Microbiology and Infectious Diseases (ESCMID) and 
Asociación Latinoamericana del Tórax (ALAT). Eur

Respir J 2017;50:1700582.

5. Ambaras Khan R, Aziz Z. The methodological quality of guidelines for hospital-acquired pneumonia and ventilatorassociated pneumonia: A systematic review. J Clin Pharm Ther 2018;43:450-9.

6. Martin-Loeches I, Rodriguez AH, Torres A. New guidelines for hospital-acquired pneumonia/ventilatorassociated pneumonia: USA vs. Europe. Curr Opin Crit Care 2018;24:347-52.

7. Madaras-Kelly KJ, Burk M, Caplinger C, et al. Total duration of antimicrobial therapy in veterans hospitalized with uncomplicated pneumonia: Results of a national medication utilization evaluation. J Hosp Med 2016;11:832-9.

8. Niederman MS, Mandell LA, Anzueto A, et al. Guidelines for the management of adults with communityacquired pneumonia. Diagnosis, assessment of severity, antimicrobial therapy, and prevention. Am J Respir Crit Care Med 2001;163:1730-54.

9. Halm EA, Fine MJ, Marrie TJ, et al. Time to clinical stability in patients hospitalized with community-acquired pneumonia: implications for practice guidelines. JAMA 1998;279:1452-7.

10. Magiorakos AP, Srinivasan A, Carey RB, et al. Multidrugresistant, extensively drug-resistant and pandrug-resistant bacteria: an international expert proposal for interim standard definitions for acquired resistance. Clin Microbiol Infect 2012;18:268-81.

11. Zhang Y, Zhong ZF, Chen SX, et al. Prevalence of healthcare-associated infections and antimicrobial use in China: Results from the 2018 point prevalence survey in 189 hospitals in Guangdong Province. Int J Infect Dis 2019;89:179-84.

12. Yu Y, Shen H, Zhu C, et al. Infections Caused by Extended-Spectrum beta-Lactamase Producing Escherichia Coli in Systemic Lupus Erythematosus Patients: Prevalence, Risk Factors, and Predictive Model. Biomed Res Int 2018;2018:8296720.

13. Aliberti S, Blasi F, Zanaboni AM, et al. Duration of

Cite this article as: Zhu C, Li Y, Yu Y, Lu L. Duration of antibiotic therapy in systemic lupus erythematosus patients with hospital-acquired bacterial pneumonia in eastern China. Ann Palliat Med 2021;10(3):2898-2906. doi: 10.21037/apm-20-584 antibiotic therapy in hospitalised patients with communityacquired pneumonia. Eur Respir J 2010;36:128-34.

14. Shen CF, Wang SM, Ho TS, et al. Clinical features of community acquired adenovirus pneumonia during the 2011 community outbreak in Southern Taiwan: role of host immune response. BMC Infect Dis 2017;17:196.

15. Cuchacovich R, Gedalia A. Pathophysiology and clinical spectrum of infections in systemic lupus erythematosus. Rheum Dis Clin North Am 2009;35:75-93.

16. Skare TL, Dagostini JS, Zanardi PI, et al. Infections and systemic lupus erythematosus. Einstein 2016;14:47-51.

17. Pappas PG, Kauffman CA, Andes DR, et al. Clinical Practice Guideline for the Management of Candidiasis: 2016 Update by the Infectious Diseases Society of America. Clin Infect Dis 2016;62:e1-50.

18. Peleg AY, Hogan DA, Mylonakis E. Medically important bacterial-fungal interactions. Nat Rev Microbiol 2010;8:340-9.

19. Roux D, Gaudry S, Khoy-Ear L, et al. Airway fungal colonization compromises the immune system allowing bacterial pneumonia to prevail. Crit Care Med 2013;41:e191-9.

20. McConnell MJ, Actis L, Pachon J. Acinetobacter baumannii: human infections, factors contributing to pathogenesis and animal models. FEMS Microbiol Rev 2013;37:130-55.

21. Cattrall JWS, Asin-Prieto E, Freeman J, et al. A pharmacokinetic-pharmacodynamic assessment of oral antibiotics for pyelonephritis. Eur J Clin Microbiol Infect Dis 2019;38:2311-21.

22. Hoff BM, Maker JH, Dager WE, et al. Antibiotic Dosing for Critically Ill Adult Patients Receiving Intermittent Hemodialysis, Prolonged Intermittent Renal Replacement Therapy, and Continuous Renal Replacement Therapy: An Update. Ann Pharmacother 2020;54:43-55.

23. Horcajada JP, Montero M, Oliver A, et al. Epidemiology and Treatment of Multidrug-Resistant and Extensively Drug-Resistant Pseudomonas aeruginosa Infections. Clin Microbiol Rev 2019;32:e00031-19. 